Regina Bendix (2009): Wenn viele Felder sich in einem Raum vereinen: Feldforschung in internationalen Gremien. In: Elfriede Hermann, Karin Klenke und Michael Dickhardt (Hg.): Form, Macht, Differenz. Motive und Felder ethnologischen Forschens. Göttingen: Universitätsverlag Göttingen, 333-346. Doi: 10.17875/gup2020-1295

\title{
Wenn viele Felder sich in einem Raum vereinen: Feldforschung in internationalen Gremien
}

\author{
Regina Bendix
}

Es ist ein sonniger Dezembertag in Genf im Jahr 2006. ${ }^{1}$ An die dreihundert Delegierte von Nationalstaaten, internationalen Organisationen, NGOs und weiteren, unterschiedlichen Gruppierungen lokaler, regionaler und überregionaler Art tröpfeln nach und nach durch die Eingangstüren des imposanten Glasbaus, der die World Intellectual Property Organization oder WIPO beherbergt. ${ }^{2}$ Die Delegierten steuern, nachdem sie sich ausgewiesen und eine Zugangsberechtigung erhalten haben, auf einen großen Sitzungssaal zu. Hier tagt zum 10. Mal das Intergovernmental Committee for Traditional Knowledge, Genetic Resources and Traditional Cultural Expressions/Folklore (IGC for TK, GR and TCE). Das Gremium trifft sich in diesem Dezember für zehn Tage statt der üblichen fünftägigen Sitzungen. Es sollen Fortschritte erzielt werden, um damit die Generalversammlung der WIPO zu überzeugen, dass das Kommissionsmandat verlängert wird. Mit Simultanübersetzungen in - wie die Anwesenden meinen - zu wenige Weltsprachen präsentieren Delegationen vorbereitete Statements, sanktioniert von ihrer Regierung oder ihrer Organisation; Dank und elaborierte Begrüßungen, Ausdruck der Hoffnung auf eine erfolgreiche Sitzung und mehr oder weniger verschlüsselte Stellungnahmen zum erwünschten Resultat sind charakteristisch für diese Interventionen $^{3}$. Spontane Diskussionen unterbrechen hin und wieder den Verlauf, bevor das Geschehen wieder zu vorbereiteten Materialien zurückkehrt.

Ambassador I Gusti Agung Wesaka Puja aus Indonesien, als Vorsitzender für diese und die letzte committee Sitzung gewählt ${ }^{4}$, bemüht sich, den oft schleppenden Fort- 
gang zu beschleunigen. In den Kaffeepausen finden sich Gruppen und Grüppchen, die sich intensiver unterhalten. Manche Teilnehmer/innen entfernen sich zielstrebig, um einer Sondersitzung einer regionalen Gruppe oder einer Interessengemeinschaft beizuwohnen. Andere bedienen sich von den massiven Stapeln ausgedruckter Dokumente in verschiedenen Sprachen am Saaleingang. Wieder andere finden sich auf dem halboffenen nächsten Stockwerk, um bei einem der Computer ihre E-Mails zu lesen oder ein eigenes Dokument auszudrucken. Das geschäftige Kommen und Gehen steht im Gegensatz zum langsamen Prozedere der eigentlichen Sitzung, die denn auch streckenweise eher dünn besucht ist. Das eigentliche Anliegen des IGCs, international akzeptable Vorschläge zur rechtlichen bis urheberrechtlichen Verankerung von Ausschnitten traditionellen Wissens, genetischer Ressourcen und traditioneller Ausdruckskraft auszuarbeiten, wird hier nämlich kaum berührt. Vielmehr häufen sich die Vorschläge dazu, wie das Prozedere in einem internationalen Verhandlungsraum wie diesem besser, gerechter, effizienter gestaltet werden könnte. Schließlich gibt der Delegierte von Japan in einer Mischung von Geduld und Verzweiflung zu bedenken: „Inhalt und Definition der Begriffe sind immer noch nicht vorhanden. Uns mangelt es an einem gemeinsamen Selbstverständnis - daher ist es zu früh, über juristisch verpflichtende Prinzipien zu sprechen." Um entscheiden zu können, ob etwas in den IP-Bereich gehöre, d.h., ob Rechte auf intellectual property oder geistiges Eigentum gefordert werden können, wären verbindliche Definitionen erforderlich. Das 16-seitige Beschlussprotokoll dieser 10. Sitzung beinhaltet denn auch einen Anhang, in welchem die zukünftig zu leistenden Arbeiten gelistet werden.

Diese japanische Intervention verdeutlicht die einer internationalen Verhandlungsrunde inhärenten und schwer zu überwindenden Hürden. Es fehlen die gemeinsame Muttersprache, die gemeinsamen Lebenskontexte und damit die gemeinsamen kommunikativen Normen. Damit ist jegliche Aushandlung verlässlicher Begriffe und Referenzrahmen ein langwieriges Unternehmen. Wer eine Sitzung des deutschen Bundestages oder des amerikanischen Kongresses für langsam hält, wird eine Sitzung innerhalb eines internationalen Verhandlungsgremiums als geradezu schleppend erleben. Für eine ethnographische Forschung dagegen birgt dieser Mangel an Tempo eine ureigene „Entdeckung der Langsamkeit“ ${ }^{\text {, }}$, die für die Erforschung internationaler Gremien und der hinter ihnen stehenden internationalen Organisationen auch eine Chance darstellt. Im vorliegenden Beitrag ist denn auch das Anliegen dieses WIPO IGCs nur von marginaler Bedeutung, insofern als die Praxen und Dynamiken internationalen Verhandelns eine hier noch dem komplexen Verhandlungsgegenstand entsprechende Typik zeigen. Zentral ist hier vielmehr die Frage, wie internationale Verhandlungsrunden als „Feld“ im Sinne einer qualitativen, kulturanthropologischen ${ }^{7}$ Forschung angegangen werden können. Hierzu soll einerseits ein Blick auf die in den letzten zwei Jahrzehnten erheblich gewachsene Literatur zu dem, was etwa anthropology of the European Union oder auch anthropology of the United Nations genannt wird, geworfen werden. Andererseits möchte ich Aufforderungen ebenso wie Berichte zur „Konstruktion des Feldes“ (Amit 
2000) auf global vernetzter Ebene auf ihr methodisches Vorgehen überprüfen, um schließlich aus den Feldforschungserfahrungen in dem eingangs geschilderten WIPO committee Vorschläge zur Festigung des methodischen Vorgehens in einem quecksilbrigen, sich in seiner Wandlungsfähigkeit dem Zugriff entwindenden Feld zu erarbeiten.

Die kulturanthropologische Erforschung internationaler Organisationen und internationaler politischer Institutionen ist in den letzten Jahrzehnten erheblich angewachsen, wenn auch viele Ergebnisse bisher noch eher auf Tagungen erprobt werden als ein Publikationsstadium erreichen. ${ }^{8}$ Die Literatur zum methodischen Vorgehen auf dieser Art von internationalem Parkett ist spärlich. Lisa Markowitz (2001) bezeichnet dieses Feld im Sinne von Laura Nader (1972) als eine Form des studying up. Dies wäre zu präzisieren, da sich seit 1972 Arbeitsfelder im Service Bereich global augenfällig verdichten und in internationaler Vernetzung ansiedeln. Sie weisen in ihrem Habitus - wenn vielleicht auch nicht in ihrem gesellschaftlichen Machtpotential - Aspekte aus der Welt der Banken und Großkorporationen auf, die Nader ins Visier genommen sehen wollte. Auch anzupassen wäre Hugh Gustersons Anmerkung "participant observation is a research technique that does not travel well up the social structure" (1997:115) - abgesehen davon, dass teilnehmende Beobachtung immer von den Statusspannungen geprägt ist, die sich unter Forschenden und Beforschten ergeben (vgl. Gullestad 2006). Es gilt vor allem, sich die Methode der „Feldforschung“ in ihrer Gänze vor Augen zu halten und darin die Komponente der teilnehmenden Beobachtung dem erweiterten Spektrum an Forschungssettings, welches die Spätmoderne birgt, anzugleichen. $U_{p}$ und down sind entsprechend Begriffe, die methodisch zu relativieren sind. Gerade der Versuch, das Wirken internationaler Organisationen nachzuvollziehen, verlangt letztendlich nach Forschungsbeziehungen, die die Vernetztheit von Akteuren in alle Richtungen wahrnimmt. Wie schwierig es Kulturanthropolog/ innen fällt, Anpassungen ihres methodischen Vorgehens an neue Felder zu akzeptieren, notierte Vered Amit, die dies dem Umstand zuschreibt, dass das Fach ein Gutteil seiner Legitimation aus der Feldforschung als Methode bezieht (2000:5).

\section{Ansätze der Feldforschung des überlokal bis international Vernetzten}

Internationales Regeln und Organisieren ist in den letzten zwei Jahrzehnten vor allem in Forschungen zur Europäischen Union, ihren Auswirkungen auf Lebenswelten, aber noch mehr ihrer Art des „Regierens“ erfasst worden, wofür auch Feldforschungen in Brüssel durchgeführt wurden (z. B. Abélès 1992, Shore 2000, 2005, Shore und Wright 1997). Nach konkreten Überlegungen dazu, wo und bei wem in Brüssel wie anzusetzen ist um welche Fragen zu beantworten, welche Rollen sich für die Feldforschenden als möglich erweisen, wie also eine Feldforschung in einem solchen Setting sich ausformuliert, sucht man vergeblich. Vielmehr kann von den verwendeten Daten her ungefähr 
zurückgeschlossen werden darauf, wie geforscht worden ist. Im Folgenden möchte ich kurz einige der Gedanken aufnehmen, die für eine umfassendere methodische Verortung in veränderten Feldern argumentiert haben, insbesondere aus den Impulsen zur multi-sited ethnography, um nach diesem Exkurs im Kontrastverfahren eine vorläufige Skizze der potentiellen methodischen Zugriffsarten anzubieten, die sich für „dichte Orte" wie das WIPO IGC eignen könnten. Es sind dies Orte, die sich in der Felderfahrung zwar als intransparent erweisen können, deren Gewebe jedoch von loser Textur bleibt, weil ein guter Prozentsatz der Akteure beständig fluktuiert. ${ }^{9}$

Internationale Entwicklungen und globale Stränge kultureller Vernetzungen sind bisher vor allem gerade in ihrer Vermehrung und damit Lösung von Lokalität untersucht worden. Einen wichtigen Anstoß zum methodischen Vorgehen auf internationalem Terrain würde man sich entsprechend von George E. Marcus' programmatischer Arbeit "Ethnography in/of the World System: The Emergence of Multi-Sited Ethnography“ (1995) erwarten. Doch der Mitorganisator jener Tagung zur Re-Evalution der ethnographischen Monographie und der methodischen Schritte, die zwischen Feldforschung und wissenschaftlicher Repräsentation liegen, die in den vieldiskutierten Band Writing Culture (Clifford und Marcus 1986) mündete, hält sich mit eigentlichen methodischen Ausführungen zur Implikation dieser Variante der Feldforschung eher zurück darüber, wie sich Feldforschung, die sich mit Fragen kultureller Verortung in globalen Vernetzungen befasst, gestalten lässt. ${ }^{10}$ Der Aufsatz zeigt letztendlich vor allem einen Fokus auf die Befindlichkeit der Forscher/innen und weniger auf die Notwendigkeit, sich auf das, was Gisela Welz (1998:177), in Anlehnung an Arjun Appadurai und Carol Breckenridge, moving targets genannt hat, einzulassen. In ihrer Anleitung, diesen Ansatz, den Appadurai (1996:52) wiederum als cosmopolitan ethnography bezeichnete, fruchtbar zu machen, kontrastiert Welz die Feldforschung in der klassischen Gemeindestudie mit einem komparativen, in mehreren Orten in parallel forschenden Ansatz und schließlich einem Ansatz, der den „Fokus von der Region selbst“ löst. Der letzte Ansatz fragt, wie Ereignisse oder Konflikte ,in sich überkreuzenden und weitgespannten antagonistischen wie kooperativen Bezügen zwischen der Region und andern Orten und Räumen auf der ganzen Welt ausgetragen wird“ (1996:190). Hierzu wird die Feldforscherin selbst mobil, um Beziehungs- und Informationsstränge sowohl räumlich wie virtuell zu verfolgen. Welz betont, dass der in der klassischen Feldforschung so wesentliche Raumbezug hier keineswegs verloren geht. Vielmehr scheint sie darauf hinzuarbeiten, mittels der Mobilität der Forscherin auch die Flexibilität des Raumes und die unterschiedlichen Dimensionen der Entgrenzung und Enträumlichung, wie sie von verschiedenen Akteuren wahrgenommen wird, zu erfassen.

$\mathrm{Zu}$ fragen ist nun, welche Erkenntnisse sich aus multi-sited Forschungsansätzen gewinnen lassen, die auch auf die lokal fokussierte Arbeit mit internationalen Gremien angewandt werden kann. Aus der wachsenden Zahl von Fallstudien, die sich als multisited ethnography begreifen, ließen sich viele herausgreifen. Zwei seien kurz erwähnt, weil sie der Komplexität von „glokalen“ Lebenssituationen und Problemen auf die Spur 
zu kommen suchen und dabei auch internationale Organisationen als Orte der Weichenstellung in den Blick nehmen. In seiner Studie zum Bau eines Riesenstaudamms in Argentinien begab sich Gustavo Ribeiro auf die Suche nach den Auswirkungen internationaler Entwicklungspolitik und transnationalen Kapitalflusses. Die Arbeit ist in ihren Schlussfolgerungen ernüchternd, insbesondere was den nachhaltigen Erfolg von grandiosen Entwicklungsprojekten betrifft. Sie ist aber methodisch aufschlussreich, insofern als Ribeiro die Gestaltung seiner multi-sited ethnography ausformuliert und dabei die Vielfalt von Institutionen und Akteuren holistisch einbezieht (1994:16). Teilnehmende Beobachtung, Interviews, Auswertung von Archivquellen und Tagespresse ergeben einen profunden Einblick in die disparaten Machtpotentiale von Wirtschaftsplanern über Architekten bis zu Material Lieferanten und deterritorialisierten Arbeitern.

Charles Briggs und Clara Mantini-Briggs verfolgten Erzählstränge unterschiedlichster Art, um den Dimensionen und Ursachen einer verheerenden Cholera Epidemie unter einer fragilen, indigenen Population in Venezuela auf den Grund zu gehen (2003). Im Fokus steht die Frage, welche Chance der Sichtbarkeit und Relevanz eine relativ kleine Bevölkerungsgruppe z. B. in einem global angelegten Gesundheitsnetzwerk sein kann. Welche Prioritätensetzungen schleichen sich in Erzählfragmente ein, die Marginalisierung letztendlich auch legitimieren? Aus Hunderten von Interviews, von der lokalen Ebene der Überlebenden bis zur World Health Organization in Genf konnten u. a. die je nach Ebene unterschiedlich gehandhabten bürokratischen Strukturen des Gesundheitswesens als Grund eruiert werden, warum eine im Prinzip heute kurierbare Seuche so viele indigene Bewohner der venezolanischen Deltas das Leben kostete.

Wie stark unterscheiden sich nun solche multi-lokalen Studien von den Anforderungen der „Feldforschung“, wie sie heute auch in vielen lokalisierten, jedoch gesellschaftlich heterogenen Settings erfordert werden? Wie nützlich sind sie als Hintergrund für die Beforschung von internationalen Organisationen als „Feld“? Beide Studien illustrieren, wie überlokal bis global angesiedelte Forschungsfragen eine differenzierende Reflektion des Begriffes "teilnehmende Beobachtung" als Haupt- oder Begleitkomponente einer Feldforschung bedingen. Brigitta Hauser-Schäublin lokalisiert die aus der teilnehmenden Beobachtung zu gewinnenden Ergebnisse aus dem „Spagat zwischen Nähe und Distanz“. Für Außenstehende ist es einfacher, „das Selbstverständliche und deshalb nahezu unsichtbar Gewordene des alltäglichen Lebens von ,normalen' Teilnehmern zu sehen. Die kulturelle Distanz ist über weite Strecken eine Voraussetzung für das Sehen “ (2003:37). Sowohl Ribeiro wie auch Briggs und Mantini Briggs kombinieren die Teilnahme in mehreren Mikrokontexten mit der Erfassung von Netzwerken, die ein räumlich nur imaginier-, nicht aber erfahrbares Forschungsfeld abgeben. Die eigene Teilhabe als Forschende an mobiler und multikontextueller Lebensweise vermittelt den notwendigen Wissensrahmen, um - in der Eruierung von funktionierenden und brüchigen Netzwerken - teilnehmend wahrnehmen zu können, 
welcher Funktionär sich der notwendigen Beziehungskaskaden von nationalem oder gar globalem Auftrag bis zur lokalen Ausführung bewusst ist, welcher regionale Krankenversorger solche Kaskaden erkennt, jedoch nicht über genügend Macht oder Autorität verfügt, sie auch durchsetzen zu können.

Trotz der limitierten "dichten Teilnahme“ (Hauser-Schäublin 2003:39-40) gibt es Konstanten methodischen Zugriffes in dieser Art der multi-sited ethnography. Feldforschende bauen auch hier soziale Beziehungen auf, die sie temporär, mit nicht zu umgehender Distanz, in die beforschten Felder einbringen. Dass die Forschenden dabei innerhalb der Beziehungs- und Strukturgeflechte, die sie beforschen, nur noch fragmentiert sichtbar sind und damit den Beforschten auch weniger Grund zur Selbstreflektion im Angesicht des Fremden geben, gälte es - in der Kontrastierung mit der klassischen, ortsgebundenen Feldstudie - zu durchdenken, genauso wie die sich oft in Bewegung befindenden Beforschten für die Feldforschenden nur in Verhaltensfragmenten erfassbar sind. Eine Konstante, die nicht die Forschenden, sondern das zu Beforschende betrifft, formuliert Sarah Strauss: In ihrer Studie zur weltweiten Verbreitung von Yoga ausgehend von einem im indischen Rishikesh gelegenen Zentrum fügte sie Marcus' mobilem Feldforscher den festen Blick auf die Konstanz der Praxis hinzu. „Die Praxis zu verfolgen“, schreibt Strauss, „,realisiert von Menschen, in Büchern und Pamphleten [...] beschrieben, in bewegten Bildern in Film und Fernsehen gefasst" (2000:181), erlaubt es, die von Marcus angedachten Verkettungen nachzuvollziehen. Für Strauss werden diese Verkettungen zu Vektoren, die zusammengefügt eine Matrix von Yoga als transnationaler Praxis ergeben. Die lokalen Praxen bleiben hiervon nicht unberührt, genauso wie die transnationale Praxis Ausschnitte des Lokalen adaptiert.

Methodisch den Vernetzungen von Menschen, Praxen und Ideen zu folgen heißt also, enträumlichte Selbstverständnisse menschlicher Verflechtung aufzudecken ebenso wie Brüche in diesem Selbstverständnis. ${ }^{11}$ Für das eingangs geschilderte IGC ist umgekehrt zu fragen, welchen Gewinn die Erforschung von „stationären“ Teilaspekten eines internationalen Zentrums, in welchem solche Netze zusammenlaufen, verspricht. Eine internationale Organisation über eine konkrete und intensive Situationsanalyse in dem spezifischen Rahmen einer Gremientagung und der darin enthaltenen Kommunikationen bietet sich hier in vieler Hinsicht als Gegenstück zur Mitverfolgung von Netzen an. Sie erlaubt eine auszugsweise Dokumentation der einfließenden und auswirkenden Netzwerke, sie dient des weiteren der Beobachtung des Lernens und Mitgestaltens seitens der beteiligten Akteure im Formieren internationaler Entscheidungsprozesse. In seinem Überblick zur Forschung über NGOs meint auch William Fisher, der selbst zu innovativen Methoden aufruft, ohne sie zu skizzieren, dass „das breite Ensemble von Netzwerken [...] nur in den chaotischen öffentlichen Spektakeln, wie sie internationale Konferenzen darstellen, greifbar" werde (1997:459). Genauso wie das Verfolgen einzelner Netzwerkstränge lohnt, wie dies Ribeiro exemplarisch getan hat, ist entsprechend auch für ein ethnographisches Einlassen auf diese temporäre Verortung zu plädieren, auch wenn sie oft wenig spektakulär sein mögen. 
Als Beispiel für ein Vortasten in die Befassung mit dieser Art von Setting kann die Forschung zu Heritage Zertifizierungsprogrammen genannt werden. ${ }^{12}$ So hat Valdimar Hafstein seine Dissertation den UNESCO Verhandlungen zur Konvention des Intangible Heritage gewidmet (2004). Sowohl als Beobachter wie auch als Mitglied einer nationalen Delegation fand er Wege zur Teilnahme in dem sich entfaltenden Rhythmus dieser Pariser Sitzungen. Gleich anderen Neuankömmlingen lernte er, wie verschiedene Sitzungsabschnitte und öffentliche Statements zu handhaben waren und erkannte die Balance zwischen diesen Aspekten und dem „Gutteil an informellem diplomatischen Manövrieren, das sich in Kaffeepausen und am Mittagstisch ebenso wie vermutlich an weiß-gedeckten Abendtafeln von Pariser Restaurants abspielte" (Hafstein 2009:99). Anne Meyer-Rath verbrachte für ihre im selben Themenfeld angesiedelte Dissertation zwei Jahre innerhalb der UNESCO Bürokratie sowie an verschiedenen Orten Afrikas, die sich um eine Weltkulturerbe Nominierung bemühten. Ihre Feldforschung zwischen Büros, Sitzungen und informellen Treffen bezeugt das temporäre Lernen internationaler Organisationsbürokratien seitens der Akteure, die sich im UNESCO Zentrum in Paris einfinden. ${ }^{13}$ In beiden Forschungsunternehmen zeigt sich, dass aus der Vielfalt von Akteuren und Intentionen, wie sie sich gebündelt im Rahmen von UNESCO Verhandlungen darstellen, durchaus Erkenntnisse gewonnen werden, die auf kulturanthropologischer Datengenese beruhen und die zu einem Teilverständnis von Globalisierungs- und Glokalisierungsprozessen beitragen. Dies soll nun im abschließenden Teil ausführlicher dargestellt werden als dies in den genannten Studien der Fall ist. Wie können die Prämissen eines Feldes, das viele Felder in sich vereint, kulturanthropologisch erkundet werden?

\section{Einlassen auf ein Hier und Jetzt}

Angesichts der Dichte potentieller Netzwerke, assoziierter Geschichte(n) und damit verbundener (Ohn-)Machtgefüge und Aspirationen mag es naiv erscheinen, die eingangs skizzierten vielen in einem Raum versammelten Felder vorerst als ein Feld in einem klar erkennbaren, zeitlich verankerten Raum zu betrachten. Doch trotz der vielen sites, die sich, je nach Fragestellung, in internationalen Organisationen als potentiell relevant eröffnen, erfährt diese Vielheit im Verhandlungssetting dennoch eine Konkretisierung in Zeit und Raum, in welcher nicht nur Begegnungen und Austausch stattfinden. Die heterogenen Akteure schaffen über den Zeitraum einer face-to-face Sitzung eine temporäre Verhandlungsgemeinschaft, die in ihrer Verschriftlichung und digitalen Disseminierung eine Verstetigung erfährt und die - nunmehr außerhalb des konkreten Ortes - Auswirkungen entlang der Netze der beteiligten Akteure haben kann. Mit anderen Worten, eine Sitzung des untersuchten WIPO IGCs ist wohl geprägt von einer Vielfalt von Kontexten, die in den Köpfen der Teilnehmer mit anreisen, doch hat auch sie Auswirkungen und hinterlässt Spuren im weiteren Geschehen in den 
repräsentierten Netzwerken. Ein forschendes Einlassen auf dieses Hier und Jetzt verschafft die Chance, die kulturellen Praxen dieses Feldes wahrzunehmen so, wie sie sich neu oder erstmalig dazustoßenden Teilnehmer/innen in diesem Setting präsentieren. Diese werden im Verlauf der Sitzungstage durch die gremiumsgewohnten ebenso wie die organisationsverantwortlichen Akteure in die Tagungsmodalitäten enkulturiert, die in eigener Beobachtung und Aneignung der Praxen ihr eigenes Agieren entfalten können.

Neuankömmlinge gibt es in Foren wie dem IGC bei der WIPO bei jeder der halbjährlichen Sitzungen sehr viele. Nur wenige der nationalen Delegationen weisen über Jahre die gleichen Gesandten auf - auf der Liste internationaler Verhandlungen liegen die Anliegen des IGCs zumindest zurzeit nicht unter den Prioritäten, so dass vor allem seitens der Industriestaaten wechselnde, oft recht junge Menschen in den Delegationssesseln Platz nehmen, die Kopfhörer für die Simultanübersetzungen aufsetzen, Papiere ausbreiten oder ihr Laptop auspacken und einschalten. Konstanter sind die Teilnehmer aus den Reihen der NGOs und anderer Beobachterorganisationen. Deren Zahl vermehrt sich jedoch beständig, so dass auch hier stets neue Partizipation zu erwarten ist. Die Position der Feldforschenden ist daher eingangs nahezu deckungsgleich mit derjenigen der Teilnehmenden - sowohl im mitgebrachten Werkzeug wie auch der weit offenen Lernhaltung.

Arbeitet man mit dem Vokabular der ethnography of speaking, so kann eine IGC Sitzung als ein mehrtätiges speech event bezeichnet werden, das neben den angereisten Delegierten auch durch die von der WIPO beschäftigen Akteure gestaltet wird und eine durch die gemeinsame Zielsetzung des Verhandelns geprägte speech community (Morgan 2004) bildet. ${ }^{14}$ Die WIPO Angestellten verleihen dem Geschehen temporäre Konstanz, sie traditionalisieren gewissermaßen manche der kommunikativen Regeln, müssen aber mit Neuerungsvorschlägen seitens der Teilnehmenden ebenso wie des von Sitzung zu Sitzung wechselnden Vorsitzes flexibel umgehen. All diese Akteure, vom distinguierten Diplomaten über die WIPO Sekretariatskraft bis zum Sicherheitspersonal, tragen Verantwortung dafür, dass dieses Ereignis organisatorisch einen gerundeten Verlauf nimmt.

Verhandeln ist die kulturelle Praxis, die im beobachteten IGC im Zentrum steht. Entsprechend verlangen die kommunikativen Verläufe im Plenum ebenso wie in kleineren mehr und weniger offiziellen Gruppensitzungen die intensivste Form teilnehmender Beobachtung. Hier gilt es, die verschiedenen Arten des Sprechens und die ihnen zukommende Gewichtung zu eruieren. Wesentliche Beachtung verdienen hier die verwendeten Codes und die Disziplinierung der verschiedenen rhetorischen Traditionen, die in einer solch internationalen Gruppe potentiell zusammentreffen. Verhandeln als eine spezifische Variante politischen Agierens weist selbstredend kulturell unterschiedliche Ausprägungen und Normen auf, die hier mit einer wenn auch flexiblen UNO-,Tradition" des angemessenen sprachlichen Auftretens zusammentreffen. Das Setting verlangt den Teilnehmern selbst multilinguale Kenntnisse ab, und die Unterstützung, 
die hierbei die Simultanübersetzung anbietet, ist selbst wieder ein konstituierendes Element des Verhandlungsambientes. Der Umgang mit Sprechen und Zuhören sowie die jeweilige Sprachwahl und der Sprachstil sind in dieser Umgebung im Vergleich zu der in der Ethnolinguistik üblicherweise ethnographierten Sprachwechselpraxis erheblich verdichtet (Woolard 2004).

Sprechen und Übersetzen sind fokussierte Akte, die seitens vieler Teilnehmer/ innen im IGC auch reflektiert werden und sich als einsteigende Interviewthematik für Leitfadeninterviews gut eignen. Ebenso wesentlich sind die Übersetzenden: Innerhalb der UNO werden sie interpreters genannt, was bereits auf die potentielle Gewichtigkeit ihrer Rolle hinweist. ${ }^{15}$ Übersetzen ist auch integraler Bestandteil der Verschriftlichung und damit Fixierung von Verhandlungsverläufen und Ergebnissen. Während die Simultanübersetzung meist mehr als drei Sprachen beinhaltet, werden IGC Dokumente online nur in Englisch, Französisch und Spanisch bereitgestellt. Zum einen reflektiert dies einen kolonial geprägten Pragmatismus: Ein Großteil der Welt erscheint linguistisch quasi abgedeckt, da die europäische Kolonisation diese Sprachen vielerorts zu Zweit- oder Amtssprachen gemacht hat. Interventionen z. B. seitens Delegierter aus China oder des arabischen Raums straft diese Annahme Lügen und der Mehraufwand, der für Anderssprachige betrieben werden muss, um sich angemessen auf die Sitzungen vorzubereiten, taucht denn auch regelmäßig unter den verhandlungstechnischen Monita auf. Die Verschriftlichung und somit die - neben dem ephemeren mündlichen Verhandeln - dauerhaftere Repräsentation der IGC Verhandlungsarbeit ist eine weitere kommunikative Dimension, durch welche das IGC Gestalt annimmt und Kontinuität sowie historische Tiefe akkumuliert. Gleichzeitig stellt die Verschriftlichung und ihre digitale Verbreitung eine Brücke in die realen Netzwerke dar, deren Vertreter/ innen die Delegierten sind. Die ausschnittweise Dokumentenanalyse, und hierbei der Vergleich zwischen eigenen Sitzungsprotokollen und den unweigerlichen Kürzungen und - wiederum - Interpretationen des Verlaufs eröffnen spannende Blicke in die Art und Weise, wie die WIPO Sekretariate ihre Gestaltungsmacht nutzen und das kokreative Moment der Sitzungen in Richtung eines im doppelten Sinne autorisierten Dokumentes verändern.

Die kommunikativen Stränge einer IGC Sitzung sind fluide und trotz gewisser gesetzter Parameter nicht rundum vorhersehbar. Diesem fluiden Aspekt des gemeinsamen Tagens steht die sachkulturell permanente Gestalt des Genfer WIPO Gebäudes mit seiner weithin sichtbaren Glasarchitektur und seiner Innenausstattung gegenüber. Materielle Gestalt wirkt sich auf den Habitus aus. Sie gibt Bewegungsmöglichkeiten und Beschränkungen vor, die körperlich-sinnlich erfahren werden. Der große Sitzungssaal mit seinen raumhohen Fenstern und den für alle Delegationen - egal wie groß das Land oder die Organisation ist, die sie vertreten - gleich großen Tischen signalisiert „demokratisches Vorgehen“ und Gleichbehandlung zumindest der Nationen, die in alphabetischer Reihenfolge nebeneinander zu sitzen haben. Die beobachtenden Organisationen finden sich zwar zusammengedrängt in den hintersten Rängen, die aber 
gleich den back benchers in manchen Nationalparlamenten auch eine bessere Übersicht genießen und am unauffälligsten den Raum verlassen können. Bisher gar nicht beachtet in Arbeiten zu internationalem Verhandeln empfiehlt sich die materielle Dimension als ein Beobachtungsmoment. Die Art, wie Delegierte und WIPO Angestellte die Räumlichkeiten nutzen, wie sie darin ihren Geschäften nachgehen, verdient Aufmerksamkeit. Die Tatsache, dass trotz digitaler Möglichkeiten zwei Mal jährlich ein direkter Austausch arrangiert wird, deutet auf die Wichtigkeit hin, die direkter Kommunikation zugestanden wird - diese findet jedoch auch immer in konkreter Verortung in Raum und Zeit statt, die genauer zu durchleuchten sich lohnt.

Eine ethnographische Zuwendung zu Orten und kommunikativen Modalitäten verspricht Ergebnisse insbesondere für das Verständnis internationalen Organisierens und Verwaltens. „Regime“ ist einer der beliebtesten Begriffe in der jüngsten kulturanthropologischen Forschung, eng verbunden mit der Erkenntnis, dass Stile der Gouvernementalität internationale bis globale Transformationen hervorbringen, die durch Regimes verfestigt werden und damit auch die vernetzten Stränge spätmodernen Lebens prägen. Sitzungen wie diejenigen des IGCs der WIPO bieten eine lokalisierte Feldforschungsgelegenheit, innerhalb welcher die Entstehung von Regimes nicht nur aber auch anhand der emergenten und flexiblen Gremienkultur dokumentiert werden kann. Das IGC ist zwar in seiner Zusammensetzung ein äußerst heterogenes Feld, in welchem in der Tat unzählige weitere potentielle Felder ihre Wege kreuzen. Es schafft aber auch - gleich anderen Gremien der Vereinten Nationen - Entscheidungsmuster, die wiederum aus dem Genfer Zentrum entlang vielfacher Netze diffundieren und so eine (sicher vielfältig adaptierte und dabei verwandelte) Prägekraft entfalten.

\section{Anmerkungen}

1 Der Beitrag beruht auf eigener Feldforschung in Genf im Dezember 2006 sowie auf den Diskussionen mit meinem Projektmitarbeiter Stefan Groth, der im Februar und von September bis November 2008 in Genf Feldforschung durchgeführt hat. Übersetzungen aus dem Englischen aus der Feldforschung ebenso wie aus der Sekundärliteratur sind von der Autorin. Für ihr Feedback zum Entwurf dieses Beitrags danke ich Brigitta Schmidt-Lauber.

2 Die WIPO wurde 1976 als eine Unterorganisation der Vereinten Nationen gegründet, vgl. WIPO < http://www.wipo.int/portal/index.html.en> [17.1.2009].

3 Intervention ist der Begriff, der hier wie in vielen andern internationalen Gremien als Bezeichnung für einen Wortbeitrag seitens einer Delegation benutzt wird.

4 Vgl. WIPO/IGC/GRTKF/10/7 Prov. 2, einsehbar unter <http://www.wipo.int/edocs/ mdocs/tk/en/wipo_grtkf_ic_10/wipo_grtkf_ic_10_7_prov_2.pdf > [8.4.2009].

5 „Content and definition of terms still not there. We lack common understanding - hence it is premature to talk about legally binding principles." Feldnotizen der Autorin vom IGC Meeting vom 30.11.-8.12.2006.

6 Der Begriff ist ausgeliehen von Romancier Sten Nadolnys Roman gleichen Titels (1983). 
7 Die Bezeichnung „Kulturanthropologie“ wird hier verwendet im angloamerikanischen Sinne der cultural anthropology, in welcher sich die vielnamigen ethnologischen Fächer des deutschen Sprachraumes zumindest partiell wiedererkennen sollten.

8 Bezeichnenderweise sind in diesem Bereich vor allem auch Forscher/innen aktiv, die in applied anthropology arbeiten. Die Zeitschrift Human Organization der Society for Applied Anthropology pupbliziert entsprechend hierzu; das Forschungsfeld Entwicklungsethnologie wäre ebenfalls als ein Bereich zu nennen, in dem die internationale Zusammenarbeit gekoppelt mit der Untersuchung internationaler Organisationen augenfällig wird. Das von Christoph Brumann und Tim Allen organisierte Panel am EASA Kongress 2008 thematisierte die "Anthropology of the United Nations“; beim Treffen der American Anthropological Association 2009 stellten George E. Marcus und Marc Abélès ihr gemeinsames Projekt „Collaborative Ethnography Inside the World Trade Organization"vor. Bei beiden Panels waren es aber vor allem Ergebnisse, nicht der methodische Zugriff, die im Mittelpunkt standen.

In anderen Fächern wie z. B. den International Relations innerhalb der Politikwissenschaft sowie der Soziologie liegt einiges an Forschungsresultaten auch zu Verhandlungsmodalitäten und deren Verwandlung vor, aber auch hier wird das Methodische kaum erwähnt.

9 Dieser Konstellation eines dichten Feldes, das von flukturierenden Akteuren belebt wird, wird im ethnographischen Schreiben auch Rechnung zu tragen sein - kann die Geertzsche Metapher der „dichten Beschreibung “ hier tragen? Dies ist eine Frage, die hier nicht weiter behandelt werden wird, die aber in der Überprüfung der mittlerweile von vielen Disziplinen übernommenen Metapher anstehen sollte.

10 Es muss hier erklärend angemerkt werden, dass die anglo-amerikanische Forschungstradition methodische Überlegungen auch in der Ausbildung marginaler behandelt hat, als dies in der deutschen Forschung üblich ist. Stockings Observers Observed (1983) ist gewissermaßen auch ein Zeugnis dafür, dass ein Bedarf bestand, sich über Traditionen der Feldforschung und damit der Datengenerierung Rechenschaft abzulegen. Die Ausbildung favorisiert zumindest in den USA oft das learning by doing, obwohl es mittlerweile Feldforschungsliteratur zu verschiedensten Feldsituationen gibt und, im Zuge der Writing Culture Wende, autobiographische Essays und Bücher zur Felderfahrung verfasst worden sind. Im deutschsprachigen Raum werden Methodenhandbücher und -lehrveranstaltungen stärker genutzt werden mit der Annahme, dass sich der wissenschaftliche Habitus des Feldforschens durchaus Schritt um Schritt vermitteln lässt. Je nach Institut und Orientierung ist hier auch augenfällig, dass auch Texte aus der empirischen Sozialforschung wie etwa der qualitativen Soziologie gerne herangezogen werden (z. B. Flick et al. 2000, Bortz und Döring 2002).

11 Intensivst diskutiert wurden die Implikationen des multi-sited Vorgehens an einer kulturanthropologischen Tagung im September 2008, wo insbesondere der Beitrag von Brigitta Schmidt-Lauber (im Druck) das stationäre und das mobile Forschen bezüglich ihres Erkenntnisgewinnes kontrastiv fokussierte.

12 Eine weitere Arena fände sich im Bereich der Ethnographie zur Verhandlung völkerrechtlicher Abkommen. Wie Merrys Überblick zur Thematik Anthropology and International Law zeigt, wird aber auch hier bisher kaum auf die methodische Positionierung der Forschenden eingegangen. Typisch scheint auch hier wieder die Problematik der ethisch kaum zu umgehenden Involvierung der forschenden Person zu sein (Merry 2006:108-109).

13 Meyer-Rath war Stipendiatin am Hamburger Institut für Sozialforschung, wo sie an einer Dissertation zum Thema „Kultur im Katalog. Zur Entstehung eines ,immateriellen Kulturerbes der Menschheit““ arbeitete. Die Information zu ihrer Feldforschung stammt aus einem unveröffentlichten Vortrag, den sie 2004 am SIEF Kongress in Marseille hielt.

14 Als Einführung zur Kommunikationsethnographie empfehlen sich Hymes (1979) sowie Bauman und Sherzer (1989). 
15 Übersetzen ist immer auch eine Interpretationsleistung, worauf Übersetzungswissenschaftler seit einiger Zeit hinweisen (Venuti 1995). Die völkerrechtliche Forschung befasst sich ebenfalls seit geraumer Zeit mit der Frage von Sprache sowohl im Verhandlungskontext wie auch in der nachfolgenden Auslegung von Rechtstexten (vgl. Schweisfurth 2006, Teil 1, Kapitel 4).

\section{Literatur}

Abélès, Marc (1992) La Vie quotidienne au Parlement européen. Paris: Hachette.

Amit, Vered, (2000) Introduction: Constructing the Field. In: Vered Amit (Hg.): Constructing the Field: Ethnographic Fieldwork in the Contemporary World, S. 1-18. London: Routledge (European Association of Social Anthropologists).

Appadurai, Arjun (1996) Modernity at Large. Cultural Dimensions of Globalization. Minneapolis: University of Minnesota Press.

Bauman, Richard und Joel Sherzer (Hg.) (1989) Explorations in the Ethnography of Speaking. Cambridge: Cambridge University Press.

Bortz, Jürgen und Nicola Döring (Hg.) (2002) Forschungsmethoden und Evaluation für Human- und Sozialwissenschaftler. Berlin: Springer.

Briggs, Charles und Clara Mantini-Briggs (2003) Stories in the Time of Cholera. Racial Profiling During a Medical Nightmare. Berkeley: University of California Press.

Fisher, William F. (1997) Doing Good? The Politics and Antipolitics of NGO Practices. Annual Review of Anthropology 26:439-464.

Flick, Uwe, Ernst von Kardoff und Ines Steinke (Hg.) (2000) Qualitative Forschung Ein Handbuch. Reinbek: Rowohlt.

Gullestad, Marianne (2006) Reconfiguring Scholarly Authority: Reflections Based on Anthropological Studies of Norway. Current Anthropology 47(6):915-932.

Gusterson, Hugh (1997) Studying Up Revisited. PoLAR: Political and Legal Anthropology Review 20(1):114-119.

Hafstein, Valdimar (2004) The Making of Intangible Cultural Heritage: Tradtion and Authenticity, Community and Humanity. Ph.D. Dissertation, University of California, Berkeley.

Hafstein, Valdimar (2009) Intangible Heritage as a List. From Masterpiece to Representation. In: Laurajane Smith und Natsuko Akagawa (Hg.): Intangible Heritage, S.93-111. London: Routledge. 
Hauser-Schäublin, Brigitta (2003). Teilnehmende Beobachtung. In: Bettina Beer (Hg.) Methoden und Techniken der Feldforschung, S. 33-54. Berlin: Reimer.

Hymes, Dell (1979) Soziolinguistik: Zur Ethnographie der Kommunikation. Frankfurt a.M.: Suhrkamp.

Markowitz, Lisa (2001) Finding the Field: Notes on the Ethnography of NGOs. Human Organization 60(1):40-46.

Marcus, George E. (1995) Ethnography in/of the World System: The Emergence of Multi-Sited Ethnography. Annual Review of Anthropology 24:95-117.

Merry, Sally Engle (2006) Anthropology and International Law. Annual Review of Anthropology 35:99-116.

Morgan, Marcyliena (2004) Speech Community. In: Alessandro Duranti (Hg.): A Companion to Linguistic Anthropology, S.3-22. Malden, Mass.: Blackwell.

Nader, Laura (1972) Up the Anthropologist - Perspectives Gained from Studying Up. In: Dell Hymes (Hg.): Reinventing Anthropology, S. 284-311. New York: Pantheon.

Nadolny, Sten (1983). Die Erfindung der Langsamkeit. München: Piper.

Ribeiro, Gustavo Lins (1994) Transnational Capitalism and Hydropolitics in Argentina: The Yacyreta High Dam. Gainesville: University Press of Florida.

Schmidt-Lauber, Brigitta (im Druck) Profil einer volkskundlich-kulturanthropologischen Empirie: Zum $(\mathrm{Zu})$ Stand der Feldforschung im Fach. In: Beate Binder und Sonja Windmüller (Hg.): Kultur - Forschung. Zum Profil einer volkskundlichen Kulturwissenschaft. Münster: Lit-Verlag.

Schweisfurth, Theodor (2006) Völkerrecht. Tübingen: Mohr Siebeck.

Shore, Cris (2000) Building Europe: The Cultural Politics of European Integration. London: Routledge.

Shore, Cris (2005) Culture and Corruption in the EU: Reflections on Fraud, Nepotism and Cronyism in the European Commission. In: Dieter Haller und Cris Shore (Hg.): Corruption: Anthropological Perspectives, S. 131-155. London: Pluto Press.

Shore, Cris und Susan Wright (Hg.) (1997) Anthropology of Policy: Critical Perspectives on Governance and Power. London: Routledge.

Stocking, George Ward Jr. (Hg.) (1983) Observers Observed: Essays on Ethnographic Fieldwork. Madison, Wisc.: University of Wisconsin Press (History of Anthropology 1). 
Strauss, Sarah (2000) Locating Yoga: Ethnography and Transnational Practice. In: Vered Amit (Hg.): Constructing the Field: Ethnographic Fieldwork in the Contemporary World, S. 162-194. London: Routledge (European Association of Social Anthropologists).

Venuti, Lawrence (1995) The Translator's Invisibility: A History of Translation. London: Routledge.

Welz, Gisela (1998) Moving Targets. Feldforschung unter Mobilitätsdruck. Zeitschrift für Volkskunde 94:177-194.

Woolard, Kathryn A. (2004) Codeswitching. In: Alessandro Duranti (Hg.): A Companion to Linguistic Anthropology, S.73-94. Malden, Mass.: Blackwell. 